\title{
Mitochondrial dysfunction in nonalcoholic fatty liver disease and alcohol related liver disease
}

\author{
Pankaj Prasun, Ilona Ginevic, Kimihiko Oishi \\ Department of Genetics and Genomic Sciences, Icahn School of Medicine at Mount Sinai, New York, NY, USA \\ Contributions: (I) Conception and design: P Prasun; (II) Administrative support: None; (III) Provision of study materials or patients: None; (IV) \\ Collection and assembly of data: None; (V) Data analysis and interpretation: None; (VI) Manuscript writing: All authors; (VII) Final approval of \\ manuscript: All authors. \\ Correspondence to: Pankaj Prasun, MD. Department of Genetics and Genomic Sciences, Icahn School of Medicine at Mount Sinai, One Gustave L. \\ Levy Place - Box 1497, New York, NY 10029, USA. Email: Pankaj.Prasun@mssm.edu.
}

\begin{abstract}
Fatty liver disease constitutes a spectrum of liver diseases which begin with simple steatosis and may progress to advance stages of steatohepatitis, cirrhosis, and hepatocellular carcinoma (HCC). The two main etiologies are-alcohol related fatty liver disease (ALD) and nonalcoholic fatty liver disease (NAFLD). NAFLD is a global health epidemic strongly associated with modern dietary habits and life-style. It is the second most common cause of chronic liver disease in the US after chronic hepatitis C virus (HCV) infection. Approximately 100 million people are affected with this condition in the US alone. Excessive intakes of calories, saturated fat and refined carbohydrates, and sedentary life style have led to explosion of this health epidemic in developing nations as well. ALD is the third most common cause of chronic liver disease in the US. Even though the predominant trigger for onset of steatosis is different in these two conditions, they share common themes in progression from steatosis to the advance stages. Oxidative stress (OS) is considered a very significant contributor to hepatocyte injury in these conditions. Mitochondrial dysfunction contributes to this OS. Role of mitochondrial dysfunction in pathogenesis of fatty liver diseases is emerging but far from completely understood. A better understanding is essential for more effective preventive and therapeutic interventions. Here, we discuss the pathogenesis and therapeutic approaches of NAFLD and ALD from a mitochondrial perspective.
\end{abstract}

Keywords: Mitochondria; nonalcoholic fatty liver disease (NAFLD); nonalcoholic steatohepatitis (NASH); metabolic syndrome; mitochondrial dysfunction

Received: 29 February 2020; Accepted: 28 April 2020; Published: 05 January 2021.

doi: $10.21037 /$ tgh-20-125

View this article at: http://dx.doi.org/10.21037/tgh-20-125

\section{Introduction}

Nonalcoholic fatty liver disease (NAFLD) is characterized by excessive hepatic fat accumulation (more than $5 \%$ of hepatocytes) in the absence of significant alcohol consumption or other secondary causes of hepatic steatosis $(1,2)$. It is not a single entity but a disease spectrum ranging from simple steatosis without inflammation, to steatosis and inflammation [nonalcoholic steatohepatitis (NASH)] with or without fibrosis, to NASH-cirrhosis, and NASHrelated hepatocellular carcinoma (HCC) $(1,3,4)$. ALD is due to heavy alcohol consumption and similar to NAFLD, its clinical presentation too varies from steatosis to more advance stages of alcoholic steatohepatitis (ASH), fibrosis, cirrhosis, and HCC (5). While, simple steatosis without inflammation is relatively benign condition and is potentially reversible, progression to steatohepatitis is associated with significant risk of further progression to cirrhosis and HCC. ASH can also present with acute hepatitis and liver failure and hence is associated with a very high mortality. Despite, significant global health burden posed by these conditions, there are very limited therapeutic options. One reason for this is lack of clear understanding of the molecular 
pathogenesis of steatosis and its progression. Oxidative stress (OS) is considered one of the significant contributors to hepatocellular injury in these conditions and a critical driver of transition from steatosis to steatohepatitis (6). Mitochondrial dysfunction is associated with NAFLD and ALD (7). As mitochondria are the main source of reactive oxygen species (ROS) generation inside a cell, mitochondrial dysfunction contributes significantly to the OS. In following sections, we examine the potential roles of mitochondrial dysfunction in steatosis and its progression to more advanced stages.

\section{Mitochondria}

\section{Mitochondria and oxidative phosphorylation (OXPHOS)}

Mitochondria are the "power house" of cell as it is the main site of energy currency (ATP) production. It is a double membranous structure. Outer mitochondrial membrane $(\mathrm{OMM})$ and inner mitochondrial membrane (IMM) are separated by intermembranous space. Glucose and fatty acids derived from food are oxidized inside mitochondria via citric acid cycle and beta oxidation, respectively. The energy stored as chemical bonds in these food constituents are released as high energy electrons by these metabolic pathways. These electrons are captured by nicotinamide adenine dinucleotide (NAD) and flavin adenine dinucleotide (FAD) leading to generation of NADH and $\mathrm{FADH}_{2}$, respectively (8). NADH and $\mathrm{FADH}_{2}$ molecules donate these high energy electrons to electron transport chain (ETC) (9). ETC is the main site of ATP generation and is located in the IMM. It consists of five complexes-complex I to V. Complex I receives electron from the NADH. Complex II accepts electrons from $\mathrm{FADH}_{2}$. Complex I and II donate electrons to coenzyme Q (CoQ). CoQ can freely diffuse through the IMM to complex III. Complex III accepts electron from the CoQ and reduces complex IV. Electrons are finally passed on to oxygen by complex IV to form water. As the electron moves along this chain, free energy is released which is used to pump protons at complexes I, III, and IV from the mitochondrial matrix to the intermembranous space establishing a proton gradient. Protons diffuse along this electrochemical gradient at complex $\mathrm{V}$ where energy released by the proton drive across the IMM is used to generate ATP from ADP (Figure 1). This process of ATP generation is also called OXPHOS. Thus, mitochondria have critical role in energy metabolism and its association with disorders of energy metabolism such as fatty liver disease appears intuitive.

\section{Mitochondrial and cellular OS}

Mitochondria are important source of reactive oxygen species (ROS) generation inside a cell $(10,11)$. ATP is generated by controlled movement of electrons along the ETC from a high energy state to low energy state in a step wise fashion. However, a very small fraction of high energy electrons (approximately 2\%) leak from the ETC and may react directly to oxygen generating superoxide radicals. Superoxide radicals are the main source of ROS inside mitochondria. Superoxide radicals are short lived and get enzymatically or spontaneously converted to hydrogen peroxide. Hydrogen peroxide may react with superoxide radical or undergo Fenton reaction to produce hydroxyl radicals $(12,13)$. In addition, superoxide radicals can react with nitrous oxide to generate peroxynitrite radicals (14). Peroxynitrite and hydroxyl radicals are highly reactive and can damage membranes, proteins, enzymes, and DNA which may result in cellular dysfunction and death. However, mitochondria also have a very efficient antioxidant mechanism. It consists of superoxide dismutase which converts superoxide radicals to hydrogen peroxide which is then converted to water by the enzymes, peroxiredoxins and glutathione peroxidase. Thus, the production of toxic hydroxyl and peroxynitrite radicals is minimized. This system is very effective and scavenges most of the ROS produced locally in the mitochondria. Mitochondria also play significant role in scavenging ROS from other cellular sources such as peroxisomes. ETC dysfunction may result in excessive electron leak and thus excessive ROS generation and cellular injury. An overproduction of ROS which exceeds cellular antioxidant defense results in damage of cellular macromolecules and alters cellular functions and viability. This is called OS (15). OS is considered the most crucial mechanism of hepatocellular injury in NASH (16). However, ROS is not always toxic. It has important intracellular messenger functions (17). Mitochondria play crucial role in maintenance of physiological level of ROS and redox hemostasis inside cells. Alterations in the redox status changes intracellular signaling and leads to metabolic reprogramming resulting in increased fat synthesis and storage (18).

\section{Mitochondria and cell death}

Pathophysiological mechanism of apoptosis consists of activation of a group of proteases called caspases (19). 


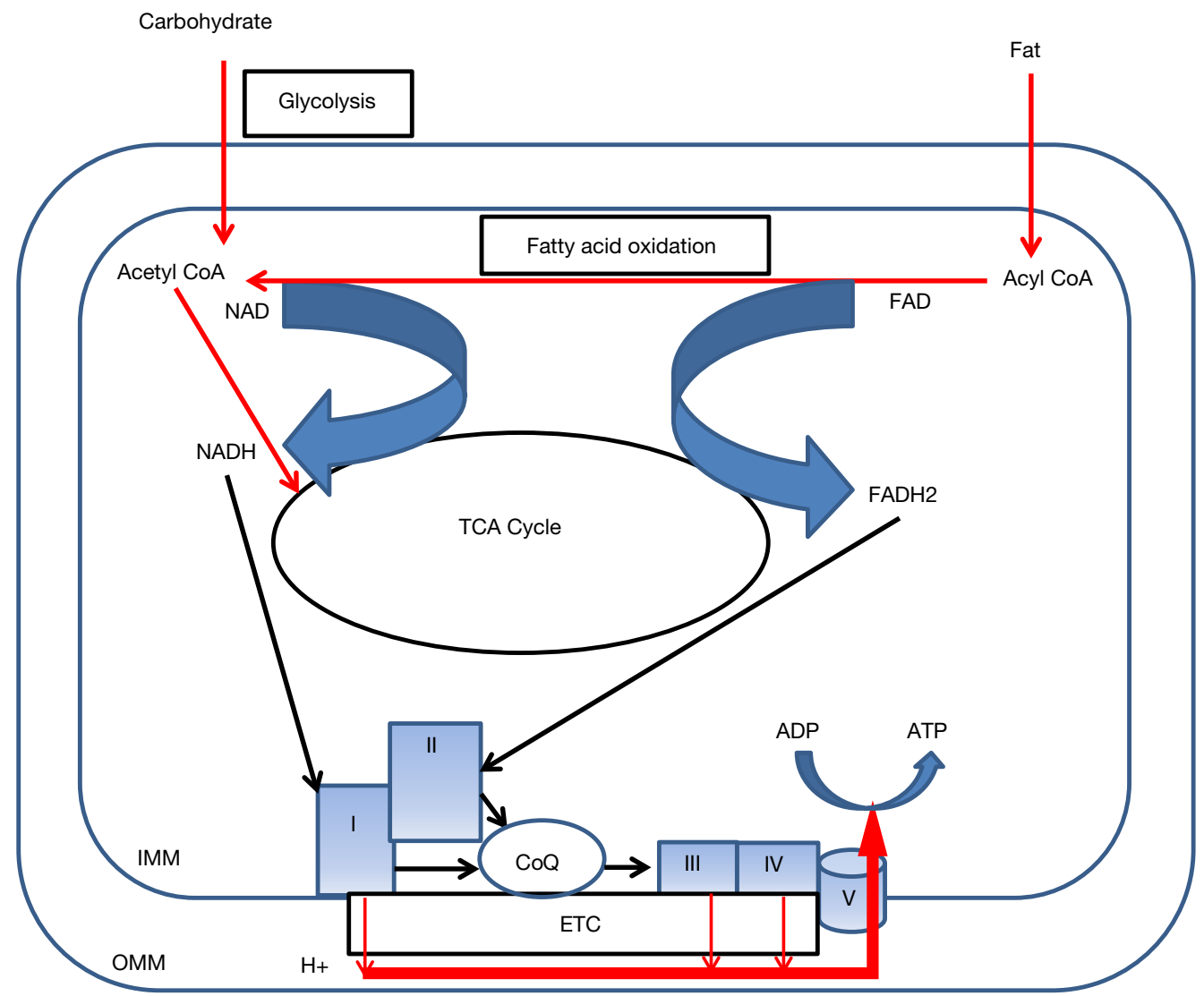

Figure 1 Diagrammatic representation of the role of mitochondria in energy metabolism inside cell. Energy stored in food constituents are captured by NAD and FAD. The high energy electrons are then transferred to ETC where it gradually moves from high energy state to low energy state. The energy released in this process is harnessed to propel protons across the IMM creating a proton gradient across the IMM. Protons then diffuse along its concentration gradient at V. The energy released in this process is harnessed to generated ATP from ADP. I, Complex I; II, Complex II; III, Complex III, IV, Complex IV; V, Complex V; CoQ, Coenzyme Q; ETC, electron transport chain; FAD, flavin adenine dinucleotide; IMM, inner mitochondrial membrane; NAD, nicotinamide adenine dinucleotide; OMM, outer mitochondrial membrane; TCA, tricarboxylic acid.

Caspases cause demolition of numerous cellular substrates leading to cell death. Apoptosis can be initiated by intrinsic and extrinsic pathways $(19,20)$. Apoptosis by intrinsic pathway can be initiated by different stimuli such as DNA damage and ROS mediated cellular injury. The ultimate outcome of these stimuli is mitochondrial outer membrane permeabilization (MOMP) and release of proapoptotic factors such as cytochrome $\mathrm{c}$ and apoptosis inducing factor (AIF) from the mitochondrial intermembranous space to the cytoplasm. Cytochrome c activates a cascade of reactions in the cytoplasm leading to caspase 3 (an executioner caspase) activation resulting in apoptosis (21). AIF localizes to nucleus and causes DNA fragmentation (22). Extrinsic pathway is initiated by binding of death signals to extracellular receptors such as Fas and TNF-related apoptosis inducing ligand (TRAIL) resulting in activation of an intracellular pathway leading to caspase 8 activation. Caspase 8 initiates apoptosis by several mechanisms including activation of caspase 3, and MOMP. Apoptosis is coordinated and energy dependent process. However, necrosis is result of energy failure. Excessive ROS injury and intramitochondrial calcium accumulation lead to creation of mitochondrial permeability transition pores (MPTP) in IMM which causes collapse of mitochondrial membrane potential, ATP depletion, mitochondrial swelling, and rapid cell death (necrosis) (23). Mitochondria play significant role in hepatocellular apoptosis and necrosis in fatty liver diseases. 


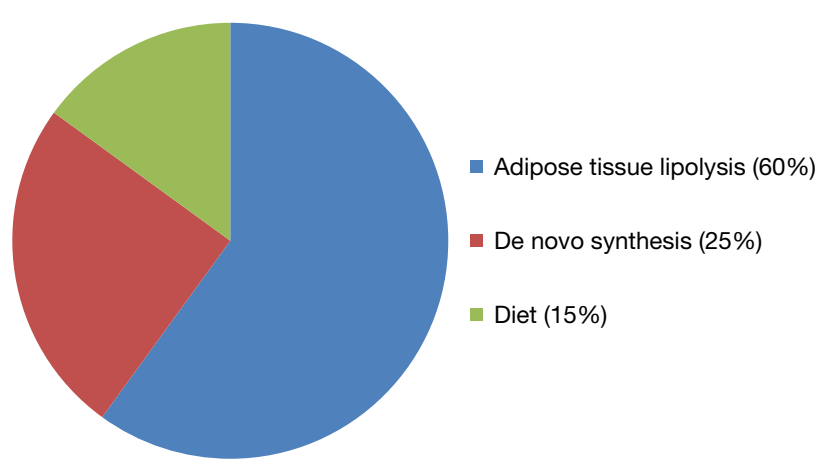

Figure 2 Origins of hepatic fat in nonalcoholic fatty liver disease.

\section{Mitochondrial biogenesis}

Mitochondria have their own DNA. However, mitochondrial DNA encodes for only a few components of the ETC and mitochondrial t-RNAs. Rest of the ETC and mitochondrial translational machinery, and other mitochondrial constituents are nuclear gene derived. Hence, mitochondrial biogenesis requires concomitant and coordinated expression of nuclear and mitochondrial genes. Peroxisome proliferatoractivated receptor gamma coactivator- $1 \alpha(\mathrm{PGC}-1 \alpha)$ is a key transcriptional activator and master regulator of mitochondrial biogenesis $(24,25)$. It regulates the process of mitochondrial biogenesis by activating several other transcription factors involved in nuclear and mitochondrial gene expression (26). The activation of transcription factors, nuclear respiratory factors 1 and 2 (NRF-1 and NRF-2), leads to induction of mitochondrial transcription factor A (TFAM) (27). TFAM directly interacts with mitochondrial genome along with mitochondrial transcription factors leading to mitochondrial gene transcription (28). Moreover, PGC- $1 \alpha$ promotes mitochondrial fatty acid oxidation by functioning as co-activator of peroxisome proliferatoractivated receptor $\alpha$ and $\delta$ (PPAR $\alpha$ and PPAR $\delta$ ), which leads to expression of mitochondrial beta fatty oxidation genes (29). Thus, PGC-1 $\alpha$ activation leads to increased mitochondrial mass and substrate oxidation. Mitochondrial biogenesis is physiological response to increased energy demand which results in increased AMP: ADP/ATP and $\mathrm{NAD}+\mathrm{NADH}$ ratios (30). PGC1 $\alpha$ activation can be caused by elevated AMP mediated by AMP activated protein kinase (AMPK), and increased NAD+ mediated by Sirtuin-1 pathways (30-32). Moreover, PGC-1 $\alpha$ activation leads to reduced cellular OS by enhanced expression of mitochondrial antioxidant enzymes such as superoxide dismutase (33). These properties of PGC- $1 \alpha$ makes it an important therapeutic target for efficient substrate handling in fatty liver diseases. Mitochondria are source and victim of OS in fatty liver disease. Damaged mitochondria are removed by a specialized autophagy called mitophagy and replaced by mitochondrial biogenesis (34). An abnormal mitophagy or impaired biogenesis leads to gradual accumulation of damaged mitochondria and depletion of healthy mitochondria thus increasing the OS and cellular dysfunction ultimately leading to cell death (35). Impairment of mitophagy and mitochondrial biogenesis plays significant role in hepatocyte injury in fatty liver diseases.

\section{Pathogenesis of fatty liver disease}

\section{Hepatic fat accumulation in NAFLD}

Excessive free fatty acid (FFA) accumulation in NAFLD is the result of increased FFA availability to hepatocytes beyond its capacity of FFA removal by oxidation or export in lipoprotein particles. The origins of FFA in hepatocytes in NAFLD are shown in Figure 2 (36).

\section{Adipose tissue in NAFLD}

Adipose tissue lipolysis is the primary driver of hepatic fat accumulation as approximately $60 \%$ of liver fat in NAFLD is adipose tissue derived. NAFLD is usually associated with surplus nutrition, obesity, and insulin resistance (IR). Adipose tissue is the reservoir for excess fat. However, adipose tissue is not merely a fat storage tissue but also an endocrine organ. It modulates metabolism by secreting adipokines (37). Hypertrophied and stressed adipocytes in NAFLD have disturbed adipokine secretory pattern. There is increase in secretion of proinflammatory adipokines such as leptin and resistin, while decrease in adiponectin. Adiponectin is an anti-inflammatory adipokine and an insulin sensitizer. In addition, local hypoxia due to adipose tissue hypertrophy and adipocyte OS due to excessive ETC activity to compensate for excessive fatty acid import in adipocytes leads to adipose tissue inflammation and infiltration by macrophages $(38,39)$. Macrophages secrete proinflammatory mediators such as interleukin-6 (IL-6) and tumor necrosis factor $\alpha(\mathrm{TNF}-\alpha)$. Thus, the imbalance between pro- and anti-inflammatory cytokines and adipokines results in local and systemic inflammation. The overall effect of altered adipokine milieu is chronic inflammation leading to IR. IR in adipose tissue leads to increased hormone sensitive lipase activity and lipolysis 


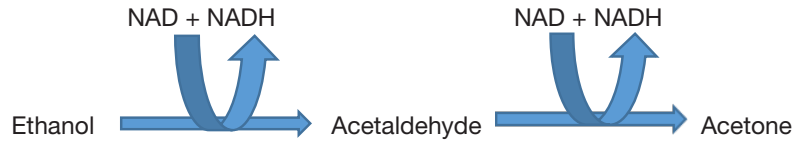

$\mathrm{ADH}($ Cytosol)

Figure 3 Primary pathway for alcohol metabolism in the hepatocytes. ADH, alcohol dehydrogenase; ALDH2, aldehyde dehydrogenase 2 .

resulting in increased plasma FFA. This excessive FFA is shunted to liver.

\section{De novo lipogenesis}

IR in peripheral tissues such as skeletal muscles leads to chronic hyperglycemia and hyperinsulinemia. IR in liver lags behind other tissues. Thus, liver is exposed to high concentrations of glucose and insulin. Hyperinsulinemia leads to increased de novo fat synthesis in liver by activation of the transcription factor, sterol regulatory binding protein-1c (SREBP-1c) (40). High glucose on the other hand stimulates lipogenesis by activation of the transcription factor, carbohydrate response element binding protein (ChREBP) (40). Progression of IR to hepatic level leads to increased hepatic gluconeogenesis and decreased glycogen synthesis. The increased hepatic glucose acts in concert with the extrahepatic glucose to activate ChREBP, thus contributing to lipogenesis.

\section{Dietary factors}

Dietary fatty acids account for about $15 \%$ of hepatic fat. Apart from dietary fat content, dietary fat composition is an important determinant of hepatic steatosis. High cholesterol stimulates hepatic lipogenesis by activation of SREBP-1c. Trans fatty acids which are often present in fast foods may increase lipogenesis by activating SREBP1c (41). Polyunsaturated fatty acids suppress SREBP-1c and thus inhibit hepatic lipogenesis (42). In addition, chronic fructose intake induces hepatic lipogenesis by activating both SREBP-1c, and ChREBP (43).

\section{Hepatic fat accumulation in ALD}

Alcohol (ethanol) is primarily metabolized in liver. In hepatocytes the main pathway for alcohol metabolism is shown in the Figure 3. Alcohol is mainly metabolized by alcohol dehydrogenase (ADH) to acetaldehyde in the cytoplasm. Acetaldehyde is a toxic molecule and highly

reactive. It can damage protein, nucleic acids, and lipids inside cell. It is rapidly metabolized to acetaldehyde by aldehyde dehydrogenase 2 (ALDH2) inside mitochondria. Both these reactions generate NADH from the NAD+ molecules thus increasing the NADH/NAD+ ratio inside cell. This changed redox status favors fatty acid synthesis and results in steatosis. In addition, alcohol oxidation also exacerbates lipogenesis by activating SREBP-1c (44). Acetaldehyde contributes to lipogenesis by inactivating PPAR $\alpha$ and thus inhibiting fatty acid oxidation (45). Chronic alcohol intake is associated with enhanced lipolysis and loss of adipose tissue mass. This enhanced flux of FFAs in liver from the adipose tissue further contributes to steatosis (46).

\section{Progression from simple steatosis to advance stages}

FFA is toxic to hepatocytes and can induce hepatocyte apoptosis by several mechanisms. It can induce hepatocyte apoptosis by both extrinsic and intrinsic pathways. FFA activates cytochrome P450 2E1 (CYP2E1) and NADPH oxidase (NOX) 4 in liver $(47,48)$. Both are oxidizing enzymes and generate free radicals. This increases OS inside hepatocyte leading to MPTP formation and hepatocyte apoptosis by release of cytochrome $\mathrm{c}$ in cytoplasm. FFA also induces transcription of proapoptotic proteins leading to MOMP. FFA and free radicals can induce endoplasmic reticulum (ER) stress and release of calcium in cytoplasm which can lead to apoptosis by activation of caspases (49). ER is closely associated with mitochondria and release of calcium at the ER-mitochondrial junction may cause increased intramitochondrial calcium and MPTP formation leading to necrosis. FFA is also toxic to lysosomes. It induces pore formation at lysosomal membrane by translocation of pore forming protein, BAX, from cytoplasm to the lysosomal membrane. Subsequently, a protease Cathepsin B is released in cytoplasm leading to activation of mitochondrial intrinsic apoptosis pathway by MOMP (50). Finally, extracellular FFA induces apoptosis by extrinsic pathway by binding to extracellular receptors involved in extrinsic apoptosis. This leads to caspase 8 activation inside hepatocyte. Caspase 8 initiates apoptosis by several mechanisms including activation of caspase 3 , and induction of MOMP (51). Thus, FFA can exert its toxic effects by several mechanisms culminating in hepatocyte death. This is also called lipotoxicity or lipoapoptosis (52). However, storage of FFA as triglyceride (TG) is protective as TG does not exert cellular damage. Hence, simple steatosis in fatty 
liver disease is considered a benign adaptive manifestation. However, when the adaptive mechanisms to contain FFA (increased mitochondrial oxidation and export from hepatocyte as lipoproteins) are overwhelmed, hepatocyte necroptosis, hepatic inflammation, and fibrosis ensue. Not all simple steatosis progress to steatohepatitis, and simple steatosis is not always a prerequisite for NASH. Genetic risk factors are considered crucial in progression of steatosis to NASH $(53,54)$.

\section{Hepatic inflammation (steatohepatitis)}

Liver inflammation and its consequences, apoptosis and fibrosis are the main determinants of poor prognosis in fatty liver diseases. NAFLD is associated with adipose tissue dysfunction as mentioned above. There is increased secretion of proinflammatory adipokines and decreased anti-inflammatory adipokines from the adipose tissue. The proinflammatory cytokines such as TNF- $\alpha$ and IL-6 act on hepatic parenchyma and promote hepatic inflammation. Gut is another source of proinflammatory materials in NAFLD (55). NAFLD is usually associated with high calorie diet rich in saturated fat and fructose. Saturated fat and fructose impair intestinal motility and disrupt intestinal mucosal barrier leading to increased release of proinflammatory dietary constituents such as FFA and fructose in circulation. In addition, intestinal dysmotility promotes dysbiosis and overgrowth of harmful bacteria. There is increased release of lipopolysaccharide (LPS) from gram negative bacterial wall to the circulation. LPS activates toll like receptor 4 (TLR4) on Kuppfer cells in hepatic sinusoids leading to secretion of proinflammatory cytokines such as IL6, IL8, and transforming growth factor $\beta$ (TGF- $\beta$ ) by Kuppfer cells, thus further promoting hepatic inflammation (56). Similar to NAFLD, there is gut bacterial overgrowth and dysbiosis in ALD (57). There is increased gut permeability for bacteria and consequent translocation to hepatic parenchyma leading to hepatic inflammation as described above. Chronic hepatic inflammation mediates hepatic insulin resistance and aggravates OS. OS is the main mechanism of hepatocyte injury and apoptosis in NASH (58).

\section{Hepatic fibrosis and HCC}

Hepatic fibrosis is due to chronic liver inflammation and hepatocyte injury. Proinflammatory cytokines particularly TGF- $\beta$ secreted by Kuppfer cells activate hepatic stellate cells (HSC) which are normally quiescent cells residing in perisinusoidal spaces (59). Once activated, they undergo proliferation and secrete collagen causing hepatic fibrosis.
Progressive hepatocyte loss leads to activation of progenitor cells. Cirrhosis is end stage liver disease characterized by replacement of much of the hepatic parenchyma by fibrotic bands separated by regenerating nodules $(60,61)$. Uncontrolled expansion of progenitor cells also predispose to HCC. However, HCC may develop with or without cirrhosis (62-64).

\section{Role of mitochondria in pathogenesis of fatty liver diseases}

OS is the main mechanism of hepatocellular injury and resulting hepatic inflammation and fibrosis in NAFLD and $\operatorname{ALD}(6,7,16,44)$. Mitochondrial dysfunction is the main contributor of OS and hence mitochondria play critical role in pathogenesis.

In NAFLD, there is increased flux of fatty acids in hepatocytes which leads to increased mitochondrial fatty acid import and oxidation. There is increased generation of $\mathrm{NADH}$ and $\mathrm{FADH}_{2}$ via fatty acid oxidation and tricarboxylic acid cycle. These molecules donate electrons to ETC where ATP is generated. However, a very small fraction of high energy electrons (approximately 2\%) leak from the ETC and may react directly to oxygen generating superoxide radicals which are the main source of ROS inside mitochondria. Thus there is increased production of ROS inside mitochondria which overwhelms the antioxidant systems culminating in OS. ROS is damaging to the mitochondrial membrane and mitochondrial DNA. ETC constituents are damaged by free radicals resulting in decline in ATP formation and enhanced leak of electrons from ETC leading to further increase in ROS generation creating a vicious cycle. OS is directly related to pathogenesis of hepatocyte death, hepatic inflammation, and fibrosis $(16,58)$.

ROS is proinflammatory as they can activate nuclear factor- $\kappa \mathrm{B}(\mathrm{NF}-\kappa \mathrm{B})$ and nucleotide-binding oligomerization domain-like receptor family, pyrin domain-containing 3 (NLRP3) inflammasome to stimulate production of inflammatory cytokines such as IL-1 $\beta$, IL-6, and TNF- $\alpha$ (65). Thus, peripheral blood monocytes and other immune mediator cells are recruited to hepatic parenchyma resulting in hepatic inflammation and further potentiation of the OS (66-68). ROS damage mitochondrial membranes and may result in MPTP formation with consequent release of mtDNA in cytoplasm which acts as danger associated molecular pattern (DAMP) and may activate NLRP3 inflammasome with consequent maturation of the cytokine, 


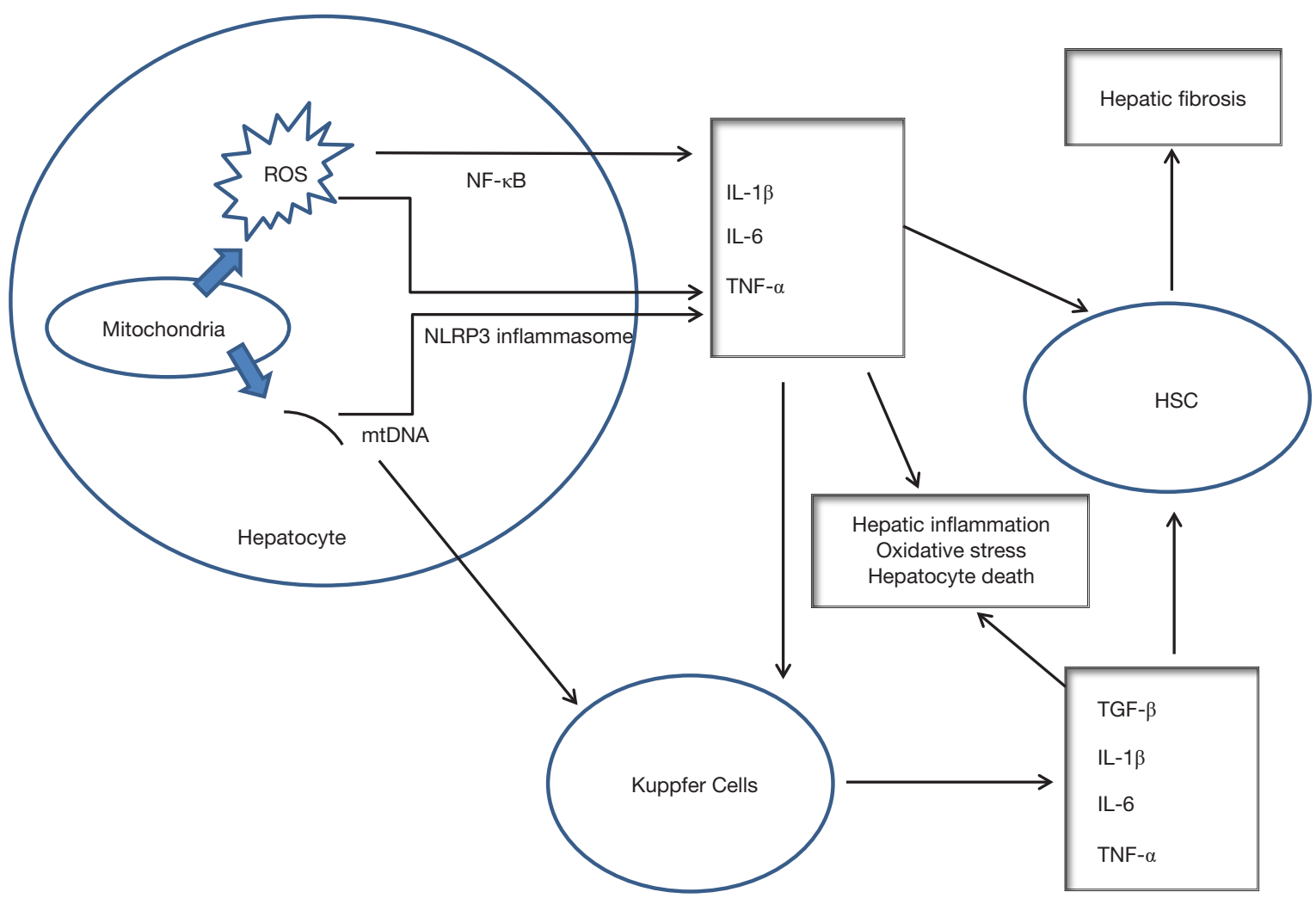

Figure 4 Schematic representation of the role of mitochondria in pathogenesis of NAFLD. There is excessive electron leak from the electron transport chain in NAFLD leading to excessive ROS and oxidative stress. The ROS activates inflammatory pathways via NF- $\kappa \mathrm{B}$ and NLRP3 pathways. The inflammatory cytokines activate Kuppfer cells in sinusoids. Mitochondrial oxidative stress also leads to MPTP formation and release of mtDNA in cytoplasm and activation of apoptosis/necrosis machinery. mtDNA activates NLRP3 inflammasome

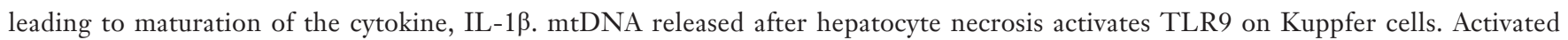
Kuppfer cells produce inflammatory cytokines and activate HSC. HSC are fibrogenic. Persistent HSC activation leads to hepatic fibrosis. The inflammatory cytokines released from hepatocytes, and innate immune cells lead to hepatic inflammation, perpetuation of oxidative stress and hepatocyte necroptosis. Loss of hepatocytes, progressive fibrosis, and regenerating nodules from progenitor cell proliferation results in cirrhosis. Uncontrolled proliferation of progenitor cells predisposes to hepatocellular carcinoma. NAFLD, nonalcoholic fatty liver disease; IL, interleukin; HSC, hepatic stellate cells; mtDNA, mitochondrial DNA; NF- $\kappa$ B, nuclear factor-Kb; NLRP3, nucleotide-binding oligomerization domain-like receptor family, pyrin domain-containing 3 ; ROS, reactive oxygen species; TGF- $\beta$, transforming growth factor- $\beta$; TNF- $\alpha$, tumor necrosis factor- $\alpha$.

IL-1 $\beta$ and perpetuation of inflammation (69). These proinflammatory cytokines also activate Kuppfer cells which then release proinflammatory cytokines potentiating the hepatic inflammation. MPTP formation leads to hepatocyte necrosis and extracellular release of mitochondrial components such as mitochondrial DNA and $\mathrm{N}$-formyl peptide which trigger activation of toll like receptor 9 (TLR9) and formyl peptide receptor 1 (FPR1) on immune mediator cells perpetuating inflammation (70-72). In addition, proinflammatory cytokines induce hepatocyte apoptosis by activating hepatocyte death receptors such as
Fas and TRAIL (73). Thus, mitochondrial dysfunction is the primary driver of hepatic inflammation and hepatocellular necroptosis. The proinflammatory cytokines secreted by hepatocyte and Kuppfer cells, particularly TGF- $\beta$ activate HSC resulting in hepatic fibrosis. Figure 4 outlines the role of mitochondrial dysfunction in NAFLD.

Mitochondrial dysfunction also plays crucial role in pathogenesis of hepatocyte injury in ALD. Apart from the cytosolic $\mathrm{ADH}$, ethanol is oxidized to acetaldehyde by cytochrome P450 2E1 (CYP2E1) in smooth ER. CYP2E1 is an inducible enzyme and its levels are elevated by chronic 
alcohol consumption. CYP2E1 induction leads to increased production of ROS such as hydroxyethyl and hydroxyl radicals leading to cellular OS (47). Moreover, the altered redox status by increased NADH/NAD+ ratio in ALD leads to enhanced formation of ferrous iron from ferric iron. Ferrous iron can undergo Fenton reaction to generate very reactive hydroxyl radicals from hydrogen peroxide. Thus, there is increased generation of ROS in hepatocytes in ALD which damages mitochondrial DNA and the ETC among other cellular constituents leading to mitochondrial DNA deletion and mitochondrial dysfunction $(74,75)$. An alcohol binge causes massive deletions in mice mitochondrial DNA (75). The damaged mitochondria are removed by mitophagy and replaced with healthy mitochondria by mitochondrial biogenesis. However, chronic alcohol consumption may lead to accumulation of mitochondrial damage and gradual depletion of mitochondria leading to mitochondrial dysfunction $(75,76)$. Mitochondrial dysfunction will further accelerate ROS generation and a vicious cycle is created. Moreover, acetaldehyde itself has proinflammatory and fibrogenic properties and is a potent mediator of chronic hepatic inflammation and fibrosis in ALD (77). Acetaldehyde produced from ethanol in gut mucosa damages gut barrier leading to increased LPS release in portal circulation and consequently hepatic Kuppfer cells activation $(77,78)$.

Loss of hepatocytes leads to compensatory proliferation of progenitor cells leading to regenerating nodules separated by fibrotic bands characteristic of cirrhosis. Proliferation of progenitor cells in the environment of chronic inflammation and OS puts them at risk of accumulating nuclear and mitochondrial mutations and consequent malignant transformation to HCC. In addition, there is activation of pro-oncogenic pathways such as c-Jun$\mathrm{N}$-terminal kinase (JNK), signal transducer and activator of transcription 3 (STAT3), and Janus kinase 2 (JAK2) pathways by the proinflammatory cytokines resulting in malignant transformation of hepatocytes $(73,79)$.

\section{Therapeutic implications}

\section{NAFLD}

NAFLD is a global health epidemic and major cause of mortality and morbidity worldwide. However, currently there are no FDA approved medications for NAFLD/ NASH. However, better understanding of molecular pathways of NAFLD pathogenesis has led to development of novel but currently investigational medications. As it is a lifestyle disease, it is not surprising that lifestyle modification is the mainstay of treatment (80). Life style modifications consist of dietary changes, exercise, and weigh loss.

\section{Dietary changes}

NAFLD is associated with hypercaloric diet rich in saturated fat, cholesterol, and refined carbohydrates. High fructose intake from excessive soft drink consumption and trans fat consumption from processed and fast foods further increases the risk of developing NAFLD $(41,42)$. Hence, dietary changes along with calorie restriction and weight loss is cornerstone of NAFLD therapy. A "high quality healthy diet" low in saturated fat, rich in monounsaturated fatty acid (MUFA) and poly unsaturated fatty acid (PUFA), and complex carbohydrates is recommended $(81,82)$. Mediterranean diet contains high proportions of vegetables, fruit, nuts, olive oil, fish, and low proportions of red meat, processed food, and refined sugar. Even though it is high in fat, it is rich in antioxidative and anti-inflammatory ingredients and hence is the recommended diet for NAFLD $(83,84)$. Olive oil is rich in monounsaturated fat while fish is rich in polyunsaturated fats, both beneficial for NAFLD. However, consumption of wine associated with Mediterranean diet should be only in moderation and individualized according to the severity of NASH. It might be altogether avoided for advance stages of NASH. In addition, a reduction or elimination of processed red meat, processed food rich in fructose, and high fructose soft drinks is recommended. Coffee drinking has favorable outcome on NAFLD. Consumption of coffee is associated with reduced risk of HCC in the setting of NASH (85). Following are the general recommendations based on current evidence (83). However, direct cause to effect relation for most of these is lacking and high quality future research is needed to substantiate.

* Follow a plant-based diet containing legumes, vegetables, and fruits which is rich in antioxidant and anti-inflammatory ingredients such as a Mediterranean diet.

* Avoid highly processed foods and soft drinks rich in fructose or high fructose corn syrup. Instead, replace processed and fast food, sweets, and commercial bakery products with whole grain and unprocessed food rich in complex carbohydrates and fiber.

* Increase consumption of PUFA and MUFA from fish, extra virgin olive oil etc. Among PUFAs, $\omega-3$ 
PUFA (found in fish oil, flaxseed, and chia seed) are more beneficial, whereas $\omega-6$ PUFA (found in canola oil, corn oil, sunflower oil) has proinflammatory properties. Hence, a higher intake of $\omega$-3 PUFAs and maintaining the $\omega-6: \omega-3$ intake ratio close to $1: 1$ is recommended $(86,87)$.

* Avoid excess alcohol consumption ( $>30 \mathrm{~g} /$ day for men and $>20$ g/day for women) $(83,88)$.

\section{Weight loss}

Although dietary changes are vital to stop the progression of NAFLD, a hypocaloric diet leading to weight loss is essential for improvement in NASH. A weight loss of $10 \%$ or more has been associated with significant improvement in liver fibrosis and inflammation in NASH (89). Hence, a weight loss of $7-10 \%$ is recommended $(80,81)$. Weight loss should be gradual and can be achieved by a combination of hypocaloric diet and exercise. However, for individuals with body mass index (BMI) of 35 or more, bariatric surgery should be considered when lifestyle interventions have failed to achieve desired outcome. After achievement of weight reduction, maintenance of reduced weight is vital. Behavioral intervention programs may be helpful in promoting healthy life style and prevention of weight regain (90). Hypocaloric diet not only prevents weight gain but also has other beneficial effects. It leads to mitochondrial biogenesis and alleviation of systemic inflammation and increases insulin sensitivity. The main molecular pathways affected by calorie restriction are:

- Sirtuin pathway: sirtuins (1 and 3) are deacetylase regulated by cellular NAD+ level. Calorie restriction leads to decline in $\mathrm{NADH}$ and consequent increased $\mathrm{NAD}+$ level. An increase in $\mathrm{NAD}+/ \mathrm{NADH}$ ratio leads to increased sirtuin 1 . Sirtuin 1 is important regulator of various intracellular processes. Increased sirtuin expression leads to increase in PGC1 $\alpha$ and hence increase in mitochondrial biogenesis and mitochondrial fat oxidation, and reduction in OS (91). Resveratrol (found in blueberry, red grape, dark chocolate) is a SIRT1 (gene for sirtuin1) activator. It has been found to improve insulin resistance associated with NAFLD $(92,93)$. Although, the evidence is lacking in humans, it has been found to ameliorate liver fibrosis and inflammation in mouse model of NASH (94).

* AMP-activated protein kinase (AMPK) pathwayAMPK acts as energy sensor and is activated by increase in AMP/ADP or AMP/ATP ratio. AMPK attempts to restore energy homeostasis by inhibiting energy consuming anabolic pathways such as lipid synthesis. In addition, it increases mitochondrial function by activation of PGC1 $\alpha$ resulting in increased mitochondrial biogenesis, fat oxidation, and anti-oxidative defense. Calorie restriction leads to increase in AMP/ATP ratio and hence activation of AMPK (95). Thiazolidinediones are antidiabetic medications which increase insulin sensitivity in skeletal muscle by AMPK activation $(96,97)$. In PIVENS trial, pioglitazone (a thiazolidinedione) was associated with improvement in hepatic inflammation and steatosis, but not in fibrosis (98). It may be considered for individuals with type 2 diabetes.

\section{Physical activity and exercise}

Sedentary life style is another independent risk factor for NAFLD. Sedentary behaviors such as excessive television watching, prolonged hours working on computer etc. increase the risk of NAFLD. Exercise, on the other hand is beneficial. Similar to calorie restriction, exercise activates AMPK and sirtuin pathways by increasing AMP/ATP and $\mathrm{NAD}+\mathrm{NADH}$ ratios, respectively. Thus, mitochondrial fat oxidation is augmented and there is reduction in OS.

\section{Antioxidant therapies}

OS is important contributor to the pathogenesis of NAFLD. Role of antioxidant therapies, both natural and artificial, is being explored in the treatment of NAFLD. Vitamin $\mathrm{E}$ has been found to be beneficial in NAFLD. It showed improvement in hepatic inflammation and steatosis, but not in fibrosis in PIVENS trial (98). Role of more potent mitochondria targeted antioxidants such as MitoQ (mitochondria targeted ubiquinone) are currently being explored as potential therapeutic agents (clinicaltrials.gov ID: NCT01167088).

\section{Other experimental therapies}

Currently there is no approved medication for NAFLD. However, few medications are under trial (Table 1). These medications target mitochondrial fatty acid oxidation and apoptosis pathways. Interestingly, mitotherapy which consists of intravenous infusion of healthy mitochondria has been tried in mice model of NAFLD and was found to be associated with reduced lipid accumulation and OS in 
Table 1 Clinical trials in nonalcoholic fatty liver disease

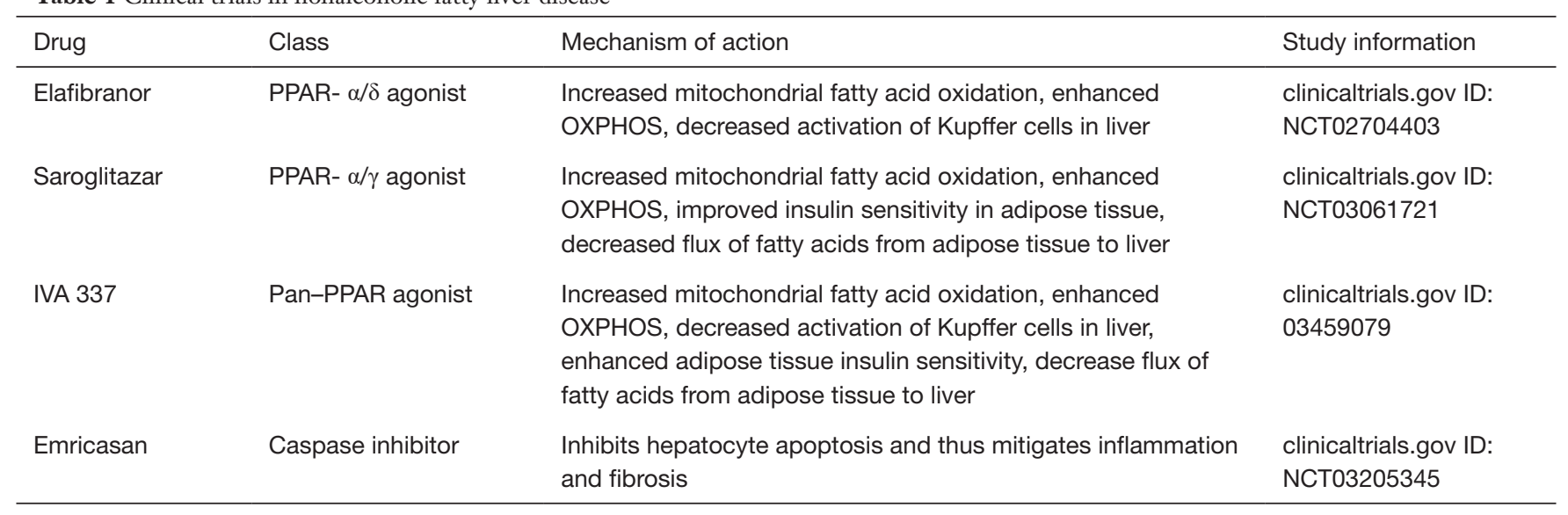

PPAR, peroxisomal proliferator-activated receptor; OXPHOS, oxidative phosphorylation.

hepatocytes and hepatocyte function was restored (99).

\section{$A L D$}

Abstinence from alcohol is the main treatment for all forms of ALD. Apart from this, anti-inflammatory medications such as steroids and infliximab (anti TNF- $\alpha$ antibody) are used in ALD. Given the role of OS in pathogenesis of ALD, several antioxidants have been tried either as monotherapy or in combination with steroids but no significant benefit was demonstrated $(100,101)$. However, replenishment of hepatic reduced glutathione (GSH) store by its precursors $\mathrm{N}$-acetyl cysteine (NAC) and S-adenosylmethionine (SAMe) were found to be of some benefit when combined with steroid $(102,103)$. Although, NAC and SAMe increase cytosolic glutathione concentration their import in mitochondria is inhibited by increased intramitochondrial cholesterol associated with ALD. Hence, a mitochondria permeable form of GSH, GSH-ethyl ester was tried and was found to be beneficial (104). Mitochondria targeted antioxidants such as MitoQ and redox nanoparticles are promising therapeutic candidates (105).

\section{Future considerations}

NAFLD and ALD are major global health problems. Current therapy is mainly preventive and effective only when implemented in early stages. However, there is lack of non-invasive biomarkers to assess the risk of progression to life threatening complications. These conditions are associated with OS not only in the liver parenchyma but also systemic inflammation and OS. Peripheral blood cells contribute to this systemic inflammation and show signs of OS and bioenergetic crisis (106). Thus, assessment of peripheral blood bioenergetic health may be an effective way to assess the systemic OS and may serve as a useful diagnostic and prognostic tool (107).

Better understanding of the molecular pathogenesis is essential for effective therapy. Mitochondrial dysfunction has emerged as one of critical mediator of hepatocyte injury in these conditions. Targeting mitochondria by diet, other lifestyle interventions, and medications provides a unique perspective for approaching these major health conditions and further research is urgently needed.

\section{Acknowledgments}

Funding: None.

\section{Footnote}

Provenance and Peer Review: This article was commissioned by the Guest Editor (Ashwani K. Singal) for the series "Non-alcoholic Fatty Liver Disease and Alcoholic Liver Disease" published in Translational Gastroenterology and Hepatology. The article was sent for external peer review organized by the Guest Editor and the editorial office.

Conflicts of Interest: All authors have completed the ICMJE uniform disclosure form (available at http://dx.doi. org/10.21037/tgh-20-125). The series "Non-alcoholic Fatty Liver Disease and Alcoholic Liver Disease" was commissioned by the editorial office without any funding or sponsorship. The authors have no other conflicts of interest 
to declare.

Ethical Statement: The authors are accountable for all aspects of the work in ensuring that questions related to the accuracy or integrity of any part of the work are appropriately investigated and resolved.

Open Access Statement: This is an Open Access article distributed in accordance with the Creative Commons Attribution-NonCommercial-NoDerivs 4.0 International License (CC BY-NC-ND 4.0), which permits the noncommercial replication and distribution of the article with the strict proviso that no changes or edits are made and the original work is properly cited (including links to both the formal publication through the relevant DOI and the license). See: https://creativecommons.org/licenses/by-nc-nd/4.0/.

\section{References}

1. Perumpail BJ, Khan MA, Yoo ER, et al. Clinical epidemiology and disease burden of nonalcoholic fatty liver disease. World J Gastroenterol 2017;23:8263-76.

2. Leoni S, Tovoli F, Napoli L, et al. Current guidelines for the management of non-alcoholic fatty liver disease: A systematic review with comparative analysis. World J Gastroenterol 2018;24:3361-73.

3. Calzadilla Bertot L, Adams LA. The Natural Course of Non-Alcoholic Fatty Liver Disease. Int J Mol Sci 2016;17:E774.

4. Vernon G, Baranova A, Younossi ZM. Systematic review: the epidemiology and natural history of non-alcoholic fatty liver disease and non-alcoholic steatohepatitis in adults. Aliment Pharmacol Ther 2011;34:274-85.

5. Singal AK, Bataller R, Ahn J, et al. ACG Clinical Guideline: Alcoholic Liver Disease. Am J Gastroenterol 2018;113:175-94.

6. García-Ruiz C, Fernández-Checa JC. Mitochondrial Oxidative Stress and Antioxidants Balance in Fatty Liver Disease. Hepatol Commun 2018;2:1425-39.

7. Mansouri A, Gattolliat CH, Asselah T. Mitochondrial Dysfunction and Signaling in Chronic Liver Diseases. Gastroenterology 2018;155:629-47.

8. Walsh CT, Tu BP, Tang Y. Eight Kinetically Stable but Thermodynamically Activated Molecules that Power Cell Metabolism. Chem Rev 2018;118:1460-94.

9. Sun F, Zhou Q, Pang X, et al. Revealing various coupling of electron transfer and proton pumping in mitochondrial respiratory chain. Curr Opin Struct Biol 2013;23:526-38.
10. Murphy MP. How mitochondria produce reactive oxygen species. Biochem J 2009;417:1-13.

11. Chen YR, Zweier JL. Cardiac mitochondria and reactive oxygen species generation. Circ Res 2014;114:524-37.

12. Pastor N, Weinstein H, Jamison E, et al. A detailed interpretation of $\mathrm{OH}$ radical footprints in a TBP-DNA complex reveals the role of dynamics in the mechanism of sequence-specific binding. J Mol Biol 2000;304:55-68.

13. Lipinski B. Hydroxyl radical and its scavengers in health and disease. Oxid Med Cell Longev 2011;2011:809696.

14. Kanaan GN, Harper ME. Cellular redox dysfunction in the development of cardiovascular diseases. Biochim Biophys Acta Gen Subj 2017;1861:2822-9.

15. Betteridge DJ. What is oxidative stress? Metabolism 2000;49:3-8.

16. Bessone F, Razori MV, Roma MG. Molecular pathways of nonalcoholic fatty liver disease development and progression. Cell Mol Life Sci 2019;76:99-128.

17. Ray PD, Huang BW, Tsuji Y. Reactive oxygen species (ROS) homeostasis and redox regulation in cellular signaling. Cell Signal 2012;24:981-90.

18. James AM, Collins Y, Logan A, et al. Mitochondrial oxidative stress and the metabolic syndrome. Trends Endocrinol Metab 2012;23:429-34.

19. Mcllwain DR, Berger T, Mak TW. Caspase functions in cell death and disease. Cold Spring Harb Perspect Biol 2013;5:a008656.

20. Parsons MJ, Green DR. Mitochondria in cell death. Essays Biochem 2010;47:99-114.

21. Lopez J, Tait SW. Mitochondrial apoptosis: killing cancer using the enemy within. Br J Cancer 2015;112:957-62.

22. Gulbins E, Dreschers S, Bock J. Role of mitochondria in apoptosis. Exp Physiol 2003;88:85-90.

23. Armstrong JA, Cash NJ, Ouyang Y, et al. Oxidative stress alters mitochondrial bioenergetics and modifies pancreatic cell death independently of cyclophilin $\mathrm{D}$, resulting in an apoptosis-to-necrosis shift. J Biol Chem 2018;293:8032-47.

24. Di W, Lv J, Jiang S, et al. PGC-1: The Energetic Regulator in Cardiac Metabolism. Curr Issues Mol Biol 2018;28:29-46.

25. Austin S, St-Pierre J. PGC1 $\alpha$ and mitochondrial metabolism--emerging concepts and relevance in ageing and neurodegenerative disorders. J Cell Sci 2012;125:4963-71.

26. Islam H, Edgett BA, Gurd BJ. Coordination of mitochondrial biogenesis by PGC- $1 \alpha$ in human skeletal muscle: A re-evaluation. Metabolism 2018;79:42-51.

27. Jornayvaz FR, Shulman GI. Regulation of mitochondrial 
biogenesis. Essays Biochem 2010; 47:69-84.

28. Barshad G, Marom S, Cohen T, et al. Mitochondrial DNA Transcription and Its Regulation: An Evolutionary Perspective. Trends Genet 2018;34:682-92.

29. Vega RB, Huss JM, Kelly DP. The coactivator PGC-1 cooperates with peroxisome proliferator-activated receptor alpha in transcriptional control of nuclear genes encoding mitochondrial fatty acid oxidation enzymes. Mol Cell Biol 2000;20:1868-76.

30. Cantó C, Auwerx J. PGC-1alpha, SIRT1 and AMPK, an energy sensing network that controls energy expenditure. Curr Opin Lipidol 2009;20:98-105.

31. Kahn BB, Alquier T, Carling D, et al. AMP-activated protein kinase: ancient energy gauge provides clues to modern understanding of metabolism. Cell Metab 2005; $1: 15-25$.

32. Dominy JE Jr, Lee Y, Gerhart-Hines Z, et al. Nutrientdependent regulation of PGC-1alpha's acetylation state and metabolic function through the enzymatic activities of Sirt1/GCN5 . Biochim Biophys Acta 2010;1804:1676-83.

33. Valle I, Alvarez-Barrientos A, Arza E, et al. PGC-1alpha regulates the mitochondrial antioxidant defense system in vascular endothelial cells. Cardiovasc Res 2005;66:562-73.

34. Yoo SM, Jung YK. A Molecular Approach to Mitophagy and Mitochondrial Dynamics. Mol Cells 2018; 41:18-26.

35. Lou G, Palikaras K, Lautrup S, et al. Mitophagy and Neuroprotection. Trends Mol Med 2020;26:8-20.

36. Donnelly KL, Smith CI, Schwarzenberg SJ, et al. Sources of fatty acids stored in liver and secreted via lipoproteins in patients with nonalcoholic fatty liver disease. J Clin Invest 2005;115:1343-51.

37. Ouchi N, Parker JL, Lugus JJ, et al. Adipokines in inflammation and metabolic disease. Nat Rev Immunol 2011;11:85-97.

38. Engin A. The Pathogenesis of Obesity-Associated Adipose Tissue Inflammation. Adv Exp Med Biol 2017;960:221-45.

39. Tateya S, Kim F, Tamori Y. Recent advances in obesityinduced inflammation and insulin resistance. Front Endocrinol (Lausanne) 2013;4:93.

40. Xu X, So JS, Park JG, et al. Transcriptional control of hepatic lipid metabolism by SREBP and ChREBP. Semin Liver Dis 2013;33:301-11.

41. Machado RM, Stefano JT, Oliveira CP, et al. Intake of trans fatty acids causes nonalcoholic steatohepatitis and reduces adipose tissue fat content. J Nutr 2010;140:1127-32.

42. Georgiadi A, Kersten S. Mechanisms of gene regulation by fatty acids. Adv Nutr 2012;3:127-34.

43. Ter Horst KW, Serlie MJ. Fructose Consumption,
Lipogenesis, and Non-Alcoholic Fatty Liver Disease. Nutrients 2017;9:E981.

44. Osna NA, Donohue TM Jr, Kharbanda KK. Alcoholic Liver Disease: Pathogenesis and Current Management. Alcohol Res 2017;38:147-61.

45. Moriya T, Naito H, Ito Y, et al. "Hypothesis of seven balances": molecular mechanisms behind alcoholic liver diseases and association with PPARalpha. J Occup Health 2009;51:391-403.

46. Zhong W, Zhao Y, Tang Y, et al. Chronic alcohol exposure stimulates adipose tissue lipolysis in mice: role of reverse triglyceride transport in the pathogenesis of alcoholic steatosis. Am J Pathol 2012;180:998-1007.

47. Leung TM, Nieto N. CYP2E1 and oxidant stress in alcoholic and non-alcoholic fatty liver disease. J Hepatol 2013;58:395-8.

48. Bettaieb A, Jiang JX, Sasaki Y, et al. Hepatocyte Nicotinamide Adenine Dinucleotide Phosphate Reduced Oxidase4 Regulates Stress Signaling, Fibrosis, and Insulin Sensitivity During Development of Steatohepatitis in Mice. Gastroenterology 2015;149:468-80.e10.

49. Cui W, Ma J, Wang X, et al. Free fatty acid induces endoplasmic reticulum stress and apoptosis of $\beta$-cells by Ca2+/calpain-2 pathways. PLoS One 2013;8:e59921.

50. Almaguel FG, Liu JW, Pacheco FJ, et al. Lipotoxicitymediated cell dysfunction and death involve lysosomal membrane permeabilization and cathepsin L activity. Brain Res 2010;1318:133-43.

51. Alkhouri N, Carter-Kent C, Feldstein AE. Apoptosis in nonalcoholic fatty liver disease: diagnostic and therapeutic implications. Expert Rev Gastroenterol Hepatol 2011;5:201-12.

52. Malhi H, Gores GJ. Molecular mechanisms of lipotoxicity in nonalcoholic fatty liver disease. Semin Liver Dis 2008;28:360-9.

53. Dongiovanni P, Anstee QM, Valenti L. Genetic predisposition in NAFLD and NASH: impact on severity of liver disease and response to treatment. Curr Pharm Des 2013;19:5219-38.

54. Sookoian S, Pirola CJ. Genetic predisposition in nonalcoholic fatty liver disease. Clin Mol Hepatol 2017;23:1-12.

55. Mokhtari Z, Gibson DL, Hekmatdoost A. Nonalcoholic Fatty Liver Disease, the Gut Microbiome, and Diet. Adv Nutr 2017;8:240-52.

56. Miura K, Ohnishi H. Role of gut microbiota and Tolllike receptors in nonalcoholic fatty liver disease. World J Gastroenterol 2014;20:7381-91. 
57. Keshavarzian A, Holmes EW, Patel M, et al. Leaky gut in alcoholic cirrhosis: a possible mechanism for alcoholinduced liver damage. Am J Gastroenterol 1999;94:200-7.

58. Masarone M, Rosato V, Dallio M, et al. Role of Oxidative Stress in Pathophysiology of Nonalcoholic Fatty Liver Disease. Oxid Med Cell Longev 2018;2018:9547613.

59. Tsuchida T, Friedman SL. Mechanisms of hepatic stellate cell activation. Nat Rev Gastroenterol Hepatol 2017;14:397-411.

60. Anstee QM, Reeves HL, Kotsiliti E, et al. From NASH to HCC: current concepts and future challenges. Nat Rev Gastroenterol Hepatol 2019;16:411-28.

61. Li B, Zhang C, Zhan YT. Nonalcoholic Fatty Liver Disease Cirrhosis: A Review of Its Epidemiology, Risk Factors, Clinical Presentation, Diagnosis, Management, and Prognosis. Can J Gastroenterol Hepatol 2018;2018:2784537.

62. Massoud O, Charlton M. Nonalcoholic Fatty Liver Disease/Nonalcoholic Steatohepatitis and Hepatocellular Carcinoma. Clin Liver Dis 2018;22:201-11.

63. Streba LA, Vere CC, Rogoveanu I, et al. Nonalcoholic fatty liver disease, metabolic risk factors, and hepatocellular carcinoma: an open question. World J Gastroenterol 2015;21:4103-10.

64. Zoller H, Tilg H. Nonalcoholic fatty liver disease and hepatocellular carcinoma. Metabolism 2016;65:1151-60.

65. Mittal M, Siddiqui MR, Tran K, et al. Reactive oxygen species in inflammation and tissue injury. Antioxid Redox Signal 2014;20:1126-67.

66. Li S, Hong M, Tan HY, et al. Insights into the Role and Interdependence of Oxidative Stress and Inflammation in Liver Diseases. Oxid Med Cell Longev 2016;2016:4234061

67. Grunhut J, Wang W, Aykut B, et al. Macrophages in Nonalcoholic Steatohepatitis: Friend or Foe? Eur Med J Hepatol 2018;6:100-9.

68. Kazankov K, Jørgensen SMD, Thomsen KL, et al. The role of macrophages in nonalcoholic fatty liver disease and nonalcoholic steatohepatitis. Nat Rev Gastroenterol Hepatol 2019;16:145-59.

69. Liu Q, Zhang D, Hu D, et al. The role of mitochondria in NLRP3 inflammasome activation. Mol Immunol 2018;103:115-24.

70. Garcia-Martinez I, Santoro N, Chen Y, et al. Hepatocyte mitochondrial DNA drives nonalcoholic steatohepatitis by activation of TLR9. J Clin Invest 2016;126:859-64.

71. Handa P, Vemulakonda A, Kowdley KV, et al. Mitochondrial DNA from hepatocytes as a ligand for TLR9: Drivers of nonalcoholic steatohepatitis? World J
Gastroenterol 2016;22:6965-71.

72. Mihm S. Danger-Associated Molecular Patterns (DAMPs): Molecular Triggers for Sterile Inflammation in the Liver. Int J Mol Sci 2018;19:E3104.

73. Kutlu O, Kaleli HN, Ozer E. Molecular Pathogenesis of Nonalcoholic Steatohepatitis- (NASH-) Related Hepatocellular Carcinoma. Can J Gastroenterol Hepatol 2018;2018:8543763.

74. Mansouri A, Fromenty B, Berson A, et al. Multiple hepatic mitochondrial DNA deletions suggest premature oxidative aging in alcoholic patients. J Hepatol 1997;27:96-102.

75. Mansouri A, Gaou I, De Kerguenec C, et al. An alcoholic binge causes massive degradation of hepatic mitochondrial DNA in mice. Gastroenterology1999;117:181-90.

76. Demeilliers C, Maisonneuve C, Grodet A, et al. Impaired adaptive resynthesis and prolonged depletion of hepatic mitochondrial DNA after repeated alcohol binges in mice. Gastroenterology 2002;123:1278-90.

77. Ceni E, Mello T, Galli A. Pathogenesis of alcoholic liver disease: role of oxidative metabolism. World J Gastroenterol 2014;20:17756-72.

78. Chaudhry KK, Samak G, Shukla PK, et al. ALDH2 Deficiency Promotes Ethanol-Induced Gut Barrier Dysfunction and Fatty Liver in Mice. Alcohol Clin Exp Res 2015;39:1465-75.

79. Léveillé M, Estall JL. Mitochondrial Dysfunction in the Transition from NASH to HCC. Metabolites 2019;9:E233.

80. Romero-Gómez M, Zelber-Sagi S, Trenell M. Treatment of NAFLD with diet, physical activity and exercise. J Hepatol 2017;67:829-46.

81. European Association for the Study of the Liver (EASL); European Association for the Study of Diabetes (EASD); European Association for the Study of Obesity (EASO). EASL-EASD-EASO Clinical Practice Guidelines for the management of non-alcoholic fatty liver disease. J Hepatol 2016;64:1388-402.

82. Eslamparast T, Tandon P, Raman M. Dietary Composition Independent of Weight Loss in the Management of NonAlcoholic Fatty Liver Disease. Nutrients 2017;9:E800.

83. George ES, Forsyth A, Itsiopoulos C, et al. Practical Dietary Recommendations for the Prevention and Management of Nonalcoholic Fatty Liver Disease in Adults. Adv Nutr 2018;9:30-40.

84. Zelber-Sagi S, Salomone F, Mlynarsky L. The Mediterranean dietary pattern as the diet of choice for non-alcoholic fatty liver disease: Evidence and plausible mechanisms. Liver Int 2017;37:936-49.

85. Gupta V, Mah XJ, Garcia MC, et al. Oily fish, coffee and 
walnuts: Dietary treatment for nonalcoholic fatty liver disease. World J Gastroenterol 2015;21:10621-35.

86. Yang J, Fernández-Galilea M, Martínez-Fernández L, et al. Oxidative Stress and Non-Alcoholic Fatty Liver Disease: Effects of Omega-3 Fatty Acid Supplementation. Nutrients 2019;11:E872.

87. Saini RK, Keum YS. Omega-3 and omega-6 polyunsaturated fatty acids: Dietary sources, metabolism, and significance - A review. Life Sci 2018;203:255-67.

88. Ratziu V, Ghabril M, Romero-Gomez M, et al. Recommendations for Management and Treatment of Nonalcoholic Steatohepatitis. Transplantation 2019;103:28-38.

89. Vilar-Gomez E, Martinez-Perez Y, Calzadilla-Bertot L, et al. Weight Loss Through Lifestyle Modification Significantly Reduces Features of Nonalcoholic Steatohepatitis. Gastroenterology 2015;149:367-78.e5.

90. Jensen MD, Ryan DH, Apovian CM, et al. 2013 AHA/ ACC/TOS guideline for the management of overweight and obesity in adults: a report of the American College of cardiology/American Heart Association Task Force on Practice Guidelines and The Obesity Society. Circulation 2014;129:S102-38.

91. Cantó C, Auwerx J. Targeting sirtuin 1 to improve metabolism: all you need is $\mathrm{NAD}(+)$ ? Pharmacol Rev 2012;64:166-87.

92. Kitada M, Koya D. SIRT1 in Type 2 Diabetes: Mechanisms and Therapeutic Potential. Diabetes Metab J 2013;37:315-25.

93. Hou CY, Tain YL, Yu HR, et al. The Effects of Resveratrol in the Treatment of Metabolic syndrome. Int J Mol Sci 2019;20:535.

94. Kessoku T, Imajo K, Honda Y, et al. Resveratrol ameliorates fibrosis and inflammation in a mouse model of nonalcoholic steatohepatitis. Sci Rep 2016;6:22251.

95. Ruderman NB, Carling D, Prentki M, et al. AMPK, insulin resistance, and the metabolic syndrome. J Clin Invest 2013;123:2764-72.

96. Coletta DK, Sriwijitkamol A, Wajcberg E, et al. Pioglitazone stimulates AMP-activated protein kinase signalling and increases the expression of genes involved

doi: $10.21037 /$ tgh-20-125

Cite this article as: Prasun P, Ginevic I, Oishi K. Mitochondrial dysfunction in nonalcoholic fatty liver disease and alcohol related liver disease. Transl Gastroenterol Hepatol 2021;6:4. in adiponectin signalling, mitochondrial function and fat oxidation in human skeletal muscle in vivo: a randomised trial. Diabetologia 2009;52:723-32.

97. Hawley SA, Gadalla AE, Olsen GS, et al. The antidiabetic drug metformin activates the AMP-activated protein kinase cascade via an adenine nucleotide-independent mechanism. Diabetes 2002;51:2420-5.

98. Sanyal AJ, Chalasani N, Kowdley KV, et al. Pioglitazone, vitamin $\mathrm{E}$, or placebo for nonalcoholic steatohepatitis. $\mathrm{N}$ Engl J Med 2010;362:1675-85.

99. Fu A, Shi X, Zhang H, et al. Mitotherapy for Fatty Liver by Intravenous Administration of Exogenous Mitochondria in Male Mice. Front Pharmacol 2017;8:241.

100.Phillips M, Curtis H, Portmann B, et al. Antioxidants versus corticosteroids in the treatment of severe alcoholic hepatitis--a randomised clinical trial. J Hepatol 2006;44:784-90.

101. Stewart S, Prince M, Bassendine M, et al. A randomized trial of antioxidant therapy alone or with corticosteroids in acute alcoholic hepatitis. J Hepatol 2007;47:277-83.

102. Nguyen-Khac E, Thevenot T, Piquet MA, et al. Glucocorticoids plus N-acetylcysteine in severe alcoholic hepatitis. N Engl J Med 2011;365:1781-9.

103. Tkachenko P, Maevskaya M, Pavlov A, et al. Prednisolone plus S-adenosil-L-methionine in severe alcoholic hepatitis. Hepatol Int 2016;10:983-7.

104.von Montfort C, Matias N, Fernandez A, et al. Mitochondrial GSH determines the toxic or therapeutic potential of superoxide scavenging in steatohepatitis. J Hepatol 2012;57:852-9.

105.Eguchi A, Yoshitomi T, Lazic M, et al. Redox nanoparticles as a novel treatment approach for inflammation and fibrosis associated with nonalcoholic steatohepatitis. Nanomedicine (Lond) 2015;10:2697-708.

106. Singal AK, Bailey SM. Cellular Abnormalities and Emerging Biomarkers in Alcohol-Associated Liver Disease. Gene Expr 2018;19:49-60.

107. Chacko BK, Kramer PA, Ravi S, et al. The Bioenergetic Health Index: a new concept in mitochondrial translational research. Clin Sci (Lond) 2014;127:367-73. 